\title{
Mittheilungen über die Hieracia des Riesengebirges.
}

Von Gustav Schneider, Bergverwalter in Schmiedeberg im Riesengebirge.

Die interessantesten Pflanzen, welche die alpine Region des Riesengebirges bewohnen, sind wohl unstreitig die Hieracia. Unter den mannigfaltigen Formen derselben verdienen namentlich diejenigen, wolche zu der Fries'schen Abtheilung der Aurella gehören, eine besondere Beachtung; nicht bloss der Mannigfaltigkeit ihrer Gestalten, sondern auch der unberechenbaren Individuenzahl wegen, in der sie im Riesengebirge auftreten. Die Alpen wie die Karpaten bieten ebenso wenig wie der Harz und andere Gebirge Deutschlands eine solche Formenmannigfaltigkeit und Individuenzahl, wio das Riesengebirge. Nur im Norden, in den schottischen und skandinavischen Gebirgen kehrt diese Mannigfaltigkeit der Formen wieder.

Die Schwierigkeit der Bestimmung aller zur Gruppe der Aurella gehörigen Hieracia, wenn sie nicht normal gestaltet sind, die vielen Irrthümer und Ungenauigkeiten in den bisherigen Publicationen veranlassen mich, eine allgemeine Uebersicht der im Riesengebirge heimischen Formen der Aurella, die ich in den letzten fünf Sommern auf 85 Hochgebirgsexcursionen zu beobachten Gelegenheit hatte, nachstehend zu veröffentlichen. Genaueres und Ausführliches werde ich später berichten, vielleicht in meiner nächstens erscheinenden Flora der Hohen Tatra.

Ich fasse, wie aus dem Folgenden hervorgeht, einzelne in Fiek's Flora von Schlesien als Varietäten beschriebenen Formen als gute Arten auf.

\section{Hieracia Aurella Fr. des Riesengebirges.}

\section{A. Hieracia Aurella alp. subfoliosa m.}

1. Hieracium alpinum L. ex p.

a) var. genuinum Tausch, Wimm. ex $\mathrm{p}$.

b) var. melanocephalum Tausch non Wimm.

Diese beiden Varietäten fasst Fiek als synonym mit $\boldsymbol{H}$. alpinum L. zusammen, sie sind aber habituell so verschieden, dass man in ihren extremsten Gestalten sie als gute Arten aufzufassen geneigt sein könnte, wenn eben keine Uebergangsformen existirten. Als stark gepresste, getrocknete Pflanzen lassen sich diese beiden Varietäten meist nur sehr schwer, öfter auch gar nicht unterscheiden, lebend sind sie aber gar nicht zu verwechseln. - Das melanocephalum hat bauchigere, oft fast kugelige Hüllen, während diese bei genuinum mehr oder weniger kreiselformig sind. Die Ligulae sind bei melanocephalum relativ kürzer und bedentend zahlreicher als bei genuinum. Aufgeblüht bilden dieselben bei genuinum eine flach ausgebreitete Scheibe, bei melanocephalum einen von dicht gedrängt stehenden Ligulis umschlossenen Trichter. Tausch schreibt letzterem gezähnte, ersterem ganzrandige Grundblätter zu; es kom- 
men aber bei beiden genannten Formen sowohl ganzrandige wie gezähnte Grundblätter - letztere allerdings bei melanocephalum am häufigsten - vor. Beide Varietäten sind im Riesengebirge gemein und wachsen meist heerdenweise beisammen. Auch in der Hohen Tatra sammelte ich beide Formen, sah aber aus den Alpen bisher nur das genuinum.

c) var. grande Wimm.

Unterscheidet sich von den vorhergehenden durch im Allgemeinen robusteren Wuchs, offter steif aufrechten, bis 0.30 M. hohen Stengel, mit $0.15 \mathrm{M}$. langen, oft zungenförmigen und meist gezähnten Grund- und mehr blattartigen - nicht bracteenförmigen Stengelblättern. Im Riesengebirge seltener, häufiger in den OstSudeten.

d) var. H. holosericeum Backh. ex p.

Im Riesengebirge kommt diese Varietät, ausgezeichnet durch dicht seidenhaarige Bekleidung und die losen, häufig blattartig auftretenden Hüllschuppen, sowohl mit kreiselförmigen, wie mit bauchigen (melonocephalum) Köpfen vor, während Backhous $\theta$ ihr involucra turbinata zuschreibt.

Als Formen, welchen die Bezeichnung Varietät nicht zukommt, sind noch zu nennen: $H$. alpinum stylosum Wimmer, $\boldsymbol{H}$. alpinum nivale Velenovsky und $H$. alp. nigrosetosum mihi.

B. Hieracia A urella al p. foliosa mihi.

2. Hieracium tubulosum Tausch., eine gute, im Riesengebirge häufige, bis $800 \mathrm{M}$. Seehőhe herabsteigende, in den Formen: a. genuinum, b. subhirsutum und c. stylosum vorkommende Art. species).

3. Hieracium montanum mihi non Jcq. (cujus planta Crepidis

Syn. H. alpinum foliosum Wimm. ex p. majore.

Var. a. Hieracium Fritzei F. Schultz ex p.

Diese Bezeichnung behalte ich für die bisher zum Wimmer'schen $H$. alpinum foliosum gerechneten Pflanzen bei mit lanzettlichen, Jänglich-oder lineal-lanzettlichen, oft zugespitzten Stengelblättern und, wenn sie zur Blüthezeit nicht abgestorben, eben solchen inneren Grundblättern. (Die äusseren Grundblätter sind bei sämmtlichen Varietäten des $H$. montanum mihi ei-spatelförmig.) Im Riesengebirge häufig.

Var. b. spathulifolium mihi ad interim.

So benenne ich die Formen des $\boldsymbol{H}$. alpinum foliosum Wimm. mit spatelförmigen inneren Grund- und Stengelblättern. Im Riesengebirge häufig, von mir auch im Felkathale der Hohen Tatra gesammelt.

Var. c. pleiocephalum Uechtr. Diesen Namen behalte ich nach Absonderung der nachfolgenden gelbgriffeligen Varietät mit Vorbehalt noch bei, bis ich über den Werth yon zwei Formen, die dann noch beim Uechtritz'schon pleiocephalum verbleiben, durch weitere Beobachtung der lebenden Pflanzen im Klaren sein werde. 
Var. d. pseudopersonatum mihi (nov. var.).

Hypophyllopodum, viride. Caulis foliosus simplex vel ex axillis foliorum subcorymbosus, pilis canis basi nigris hirsutissimus, eglandulosus. Folia basilaria in petiolum alatum pilosum longe attenuata, spathulata, pilis albis subhirsuta, ciliata (interiora subdentata, exteriora integerrima) sub anthesin emarcida, rarius persistentia; caulina sursum decrescentia, subdentata, inferiora basilaribus plus minus conformia, lanceolata, petiolata vel sessilia, superiora lanceolata sessilia, suprema bracteiformia pilis longis hirsutissima. Pedunculi hirsutissimi, pilis canis basi nigris eglandulosis. Involucra basi rotundata, pilis mollibus basi nigris sericeo hirsutissima, eglandulosa, squamis e viridi nigrescentibus, extimis latis laxis intimis acuminatis adpressis, porrectis, flores virgineos longe superantibus. Ligulae apice subciliatae, stylus in vivo luteus, in exsivcato subfuscescens vel lutescens. - Habitat in Sudetis occidentalibus (Riesengebirge) locis graminosis lapidosis haud rarum: in monte "Gehänge, ad pedem montis "Schneelcoppe" praecipue retro hospitium "Riesenbaude", ad lacum "kleiner Teich", in herbidis supra lacus "Grosser et kleiner Teich", in fauce "Melzergrube" et in monte "Kleine Sturmhaube". - Habitu accedit ad $H$. personatum Fr., sed foliis basilaribus spathulatis, sub anthesin subemarcidis, squamis extimis laxis diversum est. Ab Hieracio pleiocephalo Uechtritziano differt caule submonocephalo, semper hirsutissimo, caulinis subsessilibus, stylo luteo. Verisimile H. alpinum debile Rehmunni huic plantae affine est.

Von allen vier Varietäten des $\boldsymbol{H}$. montanum am häufigsten bei var. a. und b. kommen stylose Formen vor.

4. Hieracium eximium Backh.

Var. a. genuinum. Nur ein Exemplar fast typisch ausgebildet, von mir beim grossen Teich gefunden.

Var. b. calenduliflorum Backh. stellenweise zahlreich.

Var. c. pseudoeximium mihi ad interim.

*Hybrid. Hier. calenduliflorum $>$ decipiens Uechtr. unter den Stammeltern am Gehänge und über den Teichen.

5. Hieracium Uechtritzianum m. n. sp.

Hypophyllopodum, viride. Caulis $0.15-0.30 \mathrm{~m}$. altus, satis foliosus, monocephalus, pilis albis et setis brevibus nigris obsitus, superne glandulis sparsis immixtis. Folia subcoriacea, subhirsuta, margine ciliata; basilaria in petiolum attenuata, lanceolata vel obovata, obtusata, subintegra, sub anthesin subemarcida; caulina sursum decrescentia, lanceolata, integerrima, semiamplexicaulia vel sessilia, inferiora obtusata, saepe mucronulata, suberecta, superiora acutiuscula, erecta. Involucra basi rotundata, dense pilosa pilis mollibus eglandulosis. Squanae multiseriales latae, extimae subbracteiformes, obtusiusculae, nigricantes; intimae acuminatae, pallide virides. Ligulae intense aureae, apice subciliatae. Stylus in vivo lutescens, in exsiccato fuscescens. - Habitat in Sudetis occidentalibus (Riesengebirge) rarissimum. Locis graminosis supra convallem "Aupagrund", ad lacum "Grosser Teich", ad pedem montis "Brunnenberg", in 
monte "Krkonos". Forma stylosa ad lacum "Grosser Teich" a me lecta est. - Stirps Hieracii alpini L., Hieracio montano meo affine videtur, sed foliis subcoriaceis, latis, obtusatis, caulinis semiamplexicaulibus ab omnibus Hieraciis alpinis optime distinctum.

Nomen dedi huic plantae eximiae in honores amicissimi $\boldsymbol{R}$. v. Uechtritz Vratislaviensis, meritissimi Hieraciorum floristae.

6. Hieracium decipiens Tsch. nec Froel. nec aliorum. Syn. $H$. alpinum $\beta$. melanocephalum Wimm. non Tsch.

H. alpinum Halleri Rehm. nec Wimm., nec Rehb., nec Vill. H. nigrescens Velenovsky non Willd.

Kein Hieracium des Riesengebirges ist so vielfach falsch beurtheilt worden, als das Tausch'sche $H$. decipiens. Dieses ist eine ganz ausgezeichnete drt, die mit dem $\boldsymbol{H}$. nigrescens Willd. nur ähnliche Köpfe gemeinsam hat (squamae und Ligularbekleidung sind verschieden etc.), im Uebrigen mit demselben aber gar nicht verwandt ist. Ich bestreite, gestützt auf die Beobachtung von vielen Tausend Individuen dieser im Riesengebirge gemeinen Pflanze, das Vorkommen von Uebergangsformen zum Hier. nigrescens Willd. Bastarte kommen wohl zwischen beiden vor, aber äusserst selten. Doch darüber später Ausführlicheres. Kommt auch in der Knieholzregion der Hohen Tatra nicht selten vor. Rehmann (Oesterr. bot. Zeitschr. 1873, Nr. 3 ff.) sondert sie schon richtig von dem $\boldsymbol{H}$. nigrescens Willd. und stellt sie als subspecies zu alpinum L. vor sein H. alpinum debile.

\section{Hieracia Aurella Pseudo-Pulmonarea mihi.}

7. Hieracium nigrescens Willd. mit murorum-artigen Grundund Stengelblättern, stellenweise im Riesengebirge zahlreich.

Diese gute Art kann mit $\boldsymbol{H}$. decipiens selbst vom oberflächlichsten Beobachter nicht verwechselt werden, und darf auf keinen Fall bei diesem als Varietät einer gemeinsamen Species verbleiben. Man vergleiche übrigens die nicht zutreffenden Bemerkungen Velenovsky's in der Oesterr. bot. Zeitschr. 1883, Nr. 12, S. 386 und 387. Auf S. 388 gibt Velenovsky eine Diagnose des $\boldsymbol{H}$. decipiens Tsch. als diejenige des $H$. nigrescens Willd. (ex p., bemerkt Vel.) zum Besten.

Nach Rehmann, der in seiner Hieracienarbeit eine vortreffliche Diagnose dieser Species gibt, kommt sie auch auf den höchsten Granitgipfeln in der hohen Tatra vor.

*Hybrid. $H$. nigrescens $\times$ decipiens selten unter den Eltern.

8. Hieracium glandulosodentatum Uechtr.

Sieht manchen Formen des $\boldsymbol{H}$. vulgatum Fr. (namentlich dem vulgatim forma incisodentatum Uechtr. in sched.) habituell entfernt ähnlich, ist aber von allen anderen Hieracien sehr leicht zu unterscheiden. Steigt bis in die subalpine Region (Wiesen bei den Grenzbauden, Elbgrund) herab. 
*Hybrid: Hieracium caesium alpestre Lindbg. $\times$ glandulosodentatum Uechtr. am Kiesberge sehr selten.

Hieracium vilgatum alpestre $\times$ glandulosodentatum Uechtr. in sched. In der Melzergrube nur 1 Exemplar, aber ein gutes, von mir gefunden.

D. Hieracia Aurella Pseudo-Prenanthoidea mihi.

9. Hieracium bohemicum Fr. Auf schlesischer Seite ebenso häufig wie auf der böhmischen. Es empfiehlt sich, den vorstehenden Namen zu gebrauchen und nicht $\boldsymbol{H}$. sudeticum Stbg. ex p., wio diess in Fiek's Flora von Schlesien geschehen, um Verwechslungen mit dem folgenden, welches Stbg. ebenfalls unter seinem sudeticum verstanden hat, zu vermeiden.

10. Hieracium pedunculare Tausch syn. Hier. sudeticum der Tschechen, Stbg. ex p.

Diese distincte Art wird häufig als ein Abkömmling der vorigen durch Vermischung mit alpinum L. angesehen, was höchst unwahrscheinlich ist. Ich sah wohl Formen von $\boldsymbol{H}$. bohemicum Fr., welche in der Stellung und Form der Stengelblätter an $H$. pedunculare erinnern, noch nie aber eine Form, die zu $H$. alpinum irgend wie eine Affinität gezeigt hätte. H. montanum var. spathulifolium $\mathrm{m}$. besitzt manchmal Stengelblätter, die denen des $\boldsymbol{H}$. pedunoulare, namentlich der einköpfigen Form von Krkonoš und der Kesselkoppe ähnlich sehen, aber Köpfe, Bekleidung und der ganze Habitus sind ganz verschieden. Wer aus blossen Blattformen Uebergangsformen, namentlich nach Exsiccaten aufstellen will, wird sicher auf falsche Wege gerathen.

\title{
Beitrag zur Flora des böhmisch-mährischen und des Glatzer Schneegebirges.
}

\author{
Von Dr. Ed. Formánek, \\ k. k. Professor am böhmischen Gymnasium in Brünn.
}

(Schluss.)

Myriophyllum verticillatum L. Teltsch, Černitz, Slejboř.

- spicatum L. In den Thajaarmen bei Teltsch, Slejboř, Černitz, Krahultschy, Řasná, Neustadtl, Saar, Neu-Ingrowitz.

Eryngium campestre L. Mähr.-Budwitz otc., Ratkau ist der nächste Ort bei Teltsch, wo ich diese Art noch angetroffen habe, von da an fehlt sio oder ist höchst selten im b. G.

Sanicula europaea L. Sehr zerstreut im b. G. Rosičky und Kozinec bei Teltsch, selten bei Wlachowitz, Frischau, häufig auf der Zákowá hora, welcher Ort meilenweit von Kräutersammlern wegen dieser Pflanze, welche als volksthümliches Medicament 